\title{
Pensamiento matemático elemental y avanzado como actividad humana en permanente evolución
}

\author{
Mauricio Penagos ${ }^{a *}$, Luis Fernando Mariño ${ }^{\mathrm{b}}$, Rosa Virginia Hernández \\ ${ }^{a}$ Maestro en Ciencias en Física, Universidad Sur Colombiana Neiva, Colombia. \\ ${ }^{b}$ Estudiante de Doctorado en Educación Matemática, Universidad Francisco de Paula \\ Santander $\mathbb{D}^{\mathrm{c}}$ Magister en Educación Matemática, Universidad Francisco de Paula \\ Santander. \\ Forma de citar: Penagos, M., Mariño, L. \& Hernández, R. (2017). Mejoramiento de la competencia \\ escritural en la educación básica primaria a través de la mediación instruccional y pedagógica del video. \\ Perspectivas, 2(1). 105-116.
}

Recibido: julio 09 de 2016

Aceptado: octubre 19 de 2016

\section{Palabras clave \\ Mentes matemáticas, representación, generalización, síntesis, abstracción.}

Resumen: El escrito tiene como objeto presentar una reflexión y análisis acerca de los procesos en el desarrollo del pensamiento matemático elemental y avanzado como actividad humana. Tradicionalmente la enseñanza de la matemática tiene dos barreras por superar: Primera, que el pensamiento matemático avanzado se presenta sólo a determinada edad y por tanto debe abordarse en cursos a nivel universitario. Segunda los profesores de esta disciplina regularmente presentan la matemática a los estudiantes como un cuerpo de conocimientos acabado e irrefutable compartido por una comunidad científica. La tarea del estudiante es aprenderlo, hacer demostraciones y reproducir este conocimiento en un determinado periodo de tiempo. Entes u objetos matemáticos que en ocasiones ha tomado siglos construirlos. En contraste con el verdadero sentido de la enseñanza de esta disciplina que debe ser el de potenciar en los estudiantes el desarrollo del pensamiento matemático.

* Autor para correspondencia

mauriciopenagos@usco.edu.co

Orcid: 0000-0002-4524-2914 


\section{Keywords}

Mathematical minds, representation, generalization, synthesis, abstraction

\section{Palavras chave}

Mentes matemáticas, representação, generalização, síntese, abstração

\section{Elementary and Advanced Mathematic thinking as human activity in permanent evolution.}

Abstract :The aim of this paper is to put forward a reflection and analysis of the process surrounding the development of elementary and advanced mathematical thinking as human activity. Traditionally, mathematical teaching has had two barriers to overcome: Firstly, that advanced mathematical teaching only appears at a certain age, therefore it must be addressed in university level courses. Secondly, that the teachers of this discipline habitually present mathematics to students as a conclusive and irrefutable body of knowledge shared by a scientific community. The student's task is to learn, demonstrate and reproduce this knowledge in a specified period of time when these mathematical entities or objects have, on occasion, taken centuries to build. In contrast, the true meaning of teaching within this discipline must be that of encouraging the development of mathematical thinking in students.

\section{Pensamento Matemático primário e avançado como atividade humana na evolução permanente}

Resumo: O objetivo do trabalho é apresentar uma reflexão e análise sobre os processos no desenvolvimento do pensamento matemático elementar e avançado como atividade humana. Tradicionalmente, o ensino da matemática tem duas barreiras a superar: primeiro, o pensamento matemático avançado ocorre apenas em uma certa idade e, portanto, deve ser abordado em cursos no nível universitário. Os segundos professores desta disciplina regularmente apresentam matemática aos alunos como um corpo de conhecimento finalizado e irrefutável compartilhado por uma comunidade científica. A tarefa do aluno é aprender, demonstrar e reproduzir esse conhecimento em um determinado período de tempo. Entidades ou objetos matemáticos que, às vezes, levaram séculos para serem construídos. Em contraste com o verdadeiro significado do ensino desta disciplina que deveria ser o desenvolvimento do pensamento matemático nos estudantes. 


\section{La matemática escolar}

El conocimiento matemático hoy en día parece gozar de una aceptación social. Se considera como elemento fundamental para el desempeño de ciudadanos en un mundo globalizado. Los problemas que hoy enfrenta la sociedad varían a una tasa de cambio vertiginosa. Dificultades o problemas que enfrenta el mundo hoy día, posiblemente mañana no lo sean. Exigiendo profesionales con mentalidad abierta, generadores de conocimiento, con propuestas y soluciones creativas. El Ministerio de Educación Nacional MEN (2009), desde hace décadas viene proponiendo una serie de programas y propuestas curriculares con el propósito de mejorar la calidad de la educación. Uno de los focos centrales está relacionado con la enseñanza aprendizaje de la matemática en diferentes niveles educativos.

El camino ha sido largo y tortuoso sobre todo para profesores del área. No han interiorizado un modelo educativo proveniente de otros sistemas y países, ajeno a nuestras necesidades; cuando se les implanta uno nuevo. Se definen y redefinen objetivos, logros, indicadores de logros y estándares básicos de aprendizaje. Por último se proponen los derechos básicos de aprendizaje para la educación básica y media. La educación superior por su parte a pesar de la exigencia en cuanto a la acreditación de calidad, parece de gozar de mayor libertad.

De acuerdo con el MEN para ser matemáticamente competente, se requiere que la persona: formule, plantee y resuelva problemas. Conozca y domine el lenguaje matemático. Razone y use la argumentación, la prueba, la refutación, el ejemplo y el contraejemplo como medios para validar y/o rechazar conjeturas, avanzando hacia los procesos de la demostración. Junto al uso eficaz de procedimientos y algoritmos, entre otros. En otras palabras el objetivo primordial para los profesores de esta área debe estar enfocado en promover el desarrollo del pensamiento matemático de los estudiantes.
Por su parte el National Council of Teachers of Mathematics (NCTM, 2000) recomienda la resolución de problemas, razonamiento y prueba, comunicación, conexiones y estándares de representación para todos los programas desde prekinder hasta grado 11 o 12 según el caso. Estos estándares de proceso incluyen recomendaciones para que todos los programas de instrucción deberían permitirles a los estudiantes: (1) aplicar y adaptar una variedad de estrategias apropiadas para resolver problemas; (2) seleccionar y usar varios tipos de razonamiento y métodos de prueba; (3) analizar y evaluar el pensamiento matemático propio y las estrategias de otros; (4) entender cómo las ideas matemáticas se interconectan; y (5) seleccionar, aplicar y traducir entre representaciones matemáticas para resolver problemas.

En contraste a todo esto, la realidad es diferente: pareciera que la situación ha desmejorado respecto al pasado. Sí de esto se trata basta con mirar el bajo desempeño de los estudiantes en diferentes pruebas tanto a nivel nacional como internacional.

En los primeros años de escuela a los niños les llama la atención y a su manera construyen conocimientos matemáticos. Así no los exterioricen por supuesto. A medida que transcurre el tiempo el interés parece ir desapareciendo, cuando llegan a su juventud y tienen que escoger un programa académico para su formación profesional en el cual se van a desempeñar durante toda su vida, un alto porcentaje de ellos prefiere carreras que no tengan relación con la matemáticas y menos aún si de matemática pura se trata.

Incluso algunos de ellos optan por programas de ingeniería. Pero parece ser también que la gran cantidad de contenidos matemáticos y su complejidad son una posible causa de deserción. Hay excepciones por supuesto con niños que tienen capacidades innatas para ésta disciplina. Camarena (2009) manifiesta que es reconocida en el contexto nacional e internacional la problemática que enfrentan los estudiantes en el aprendizaje de la matemática en los diferentes niveles educativos. Y "se puede decir que la gran mayoría del alumnado 
no tiene claro por qué estudia matemáticas, lo cual demerita la motivación hacia esta ciencia” (p.16). Esta misma autora reitera que los factores pueden ser de diversa índole: “de tipo social, económico, de orden curricular, asociados a la didáctica, inherentes a los docentes, el tema de estudio o causas de infraestructura cognoscitiva de los alumnos" (p. 1).

Al momento del iniciar un periodo académico en cualquier nivel educativo hay una preocupación compartida por los profesores de esta disciplina: tener que planificar, organizar y reorganizar actividades para impartir una gran cantidad de contenidos en un tiempo determinado. A pesar de ello, se planean y proponen actividades a lo largo del periodo que permitan cubrir la totalidad de contenidos, presentado las matemáticas a los estudiantes como un saber acabado y pulido; como producto conocido e irrefutable, plenamente aceptada y reconocido por la comunidad matemática (Dreyfus, 2002 ).

Procesos de descubrir o redescubrir las relaciones en objetos matemáticos o entre ellos, a menudo son considerados como uno de los medios más eficaces para que los niños aprendan matemáticas. Sin embargo, consumen gran cantidad de tiempo. Esto parece ser razón suficiente para qué profesores, especialmente los de matemáticas más avanzadas, tienden a no utilizar estas estrategias pues no permiten abordar la totalidad de contenidos planeados para un lapso de tiempo.

\section{Diferentes tipos de mentes matemáticas}

Desde hace varias décadas es reconocida la dificultad fundamental acerca de la discusión de la naturaleza de la psicología del pensamiento matemático avanzado (PMA). La materia envuelve dos disciplinas: psicología y matemáticas. El ideal sería que una persona fuera psicólogo y matemático al mismo tiempo. Debido a la falta de expertos con estas características y según la tradición; la materia debe ser investigada por matemáticos en un lado y por psicólogos en otro.
Cada exponente está interesado en ver el PMA desde su propia perspectiva. Los psicólogos posiblemente para extender la teoría de la psicología, conocer y pensar procesos más complejos de dominio de la sabiduría. Los matemáticos para buscar percepción dentro de los procesos de pensamiento creativo, quizás con la esperanza de mejorar la calidad de la enseñanza o investigación.

Para Poincaré (1913), citado por Tall (2002) “es imposible estudiar el trabajo del gran matemático o de quienes lo siguen sin darse cuenta de las dos tendencias contrarias o preferibles de las dos clases de diferencias de pensamiento" (p. 4). Según Poincaré existen muchos tipos de pensamiento matemático. Por ejemplo Kronecker y su visión intuicionista afirma que los conceptos matemáticos sólo existen cuando su construcción se demuestra a partir del dominio de los enteros. Mientras que la visión lógica de Russell declara que las matemáticas consisten en deducciones utilizando las leyes de la lógica. Entretanto Hilbert y su visión formalista acerca de que la matemática es la manipulación significativa de marcas sin sentido escrita en papel.

Por su parte Tall (2002) hace un llamado y afirma que: "cualquier teoría de la psicología del aprendizaje de las matemáticas debe tener en cuenta no sólo las crecientes concepciones de los estudiantes, sino las concepciones de los matemáticos maduros" (p. 5), y que todos tenemos maneras sutilmente diferentes de ver un concepto matemático dado, dependiendo de nuestras experiencias anteriores. Para Poincaré citado en Tall (2002 ), "algunos prefieren tratar sus problemas «por análisis», otros "por geometría”. Los primeros son incapaces de "ver en el espacio", los demás se cansan rápidamente de largos cálculos y se quedan perplejos (p. 4).

Uno de los principales objetivos de los profesores de matemáticas es la comprensión. ¿Pero qué es la comprensión?, ¿Cómo sucede?. Para Dreyfus (2002) "es un proceso que ocurre en la mente del estudiante y se basa en una larga secuencia de actividades de aprendizaje durante el cual una gran variedad de procesos mentales ocurren 
e interactuan" (p. 25). Por otro lado investigadores en psicología como Marrón, Bransford, Ferrara, y Campione citados en Dreyfus (2002) se preguntan: ¿qué significa conocimiento?, ¿Cuáles son sus componentes?, ¿Qué procesos mentales pueden intervenir y se combinan para formar esa metaproceso de comprensión?. Responder estos cuestionamientos no es fácil aunque profesores e investigadores se han ingeniado diferentes estrategias que finalmente son muy poco confiables puesto que esto ocurre en la mente de las personas.

Para Tall (2002) cualquier teoría de la psicología del pensamiento matemático debe ser vista en el más amplio contexto de la actividad mental y cultural humana. No existe una verdad absoluta, para pensar sobre matemáticas, pero si hay diversas formas desarrolladas culturalmente de pensamiento en los cuales varios aspectos son relativos al contexto.

\section{Pensamiento matemático elemental y pensamiento matemático avanzado}

El pensamiento matemático puede analizarse desde diversas perspectivas y enfoques. Lo que intentamos mostrar en el documento son algunos aspectos referentes al desarrollo del pensamiento matemático elemental o avanzado como procesos cognitivos que ocurre en la mente de las personas.

$\mathrm{Al}$ intentar hablar de pensamiento matemático avanzado, lo primero que viene a la mente es: ¿Qué es o qué se entiende por pensamiento matemático avanzado (PMA)?, ¿Existen diferencias claras entre pensamiento matemático elemental (PME) y (PMA)? Estudiosos como Sternberg (1996) afirman que no existe un acuerdo sobre que es el Pensamiento Matemático.

Selden y Selden. (2013) afirman que el término PMA ha estado cargado de ambigüedad. Estos autores se preguntan: ¿el término avanzado se refiere a las matemáticas, al pensamiento o a ambos? ¿Qué tipos especiales de pensamiento en estudiantes universitarios, estudiantes graduados y matemáticos están especialmente asociados con las matemáticas avanzadas? De hecho, ¿qué tipos de matemática se podrían considerar razonablemente avanzada desde el prekinder hasta los planes de estudios de posgrado?

Claramente, los temas más avanzados, como el cálculo o las ecuaciones diferenciales, no se pueden comprender sin una comprensión sólida de temas más elementales, como la función y la tasa de cambio. Por su parte para Dreyfus (1990) el pensamiento se basa en un continuo: procesos como el análisis, la conjetura, la definición, la formalización, la demostración, la generalización y la síntesis, aunque más frecuentes en las matemáticas más avanzadas, pueden y deben desarrollarse desde niveles elementales en adelante.

Según Dreyfus (2002) "no hay una clara distinción entre muchos de los procesos de enseñanza elemental y matemática avanzada, aunque las matemáticas avanzadas se centran más en las abstracciones de la definición y la deducción" ( $p$. 26). Algunos de estos procesos son o los relacionan con el nivel de enseñanza de la matemática, algunos con la enseñanza en la escuela y otros con la universitaria. Para Dreyfus es posible pensar temas relacionados con la matemática avanzada de forma elemental. Estos pueden estar presentes en niños de corta edad. Por ejemplo cuando los niños se enfrentan a problemas de olimpiadas matemáticas se evidencian estas características.

En 1985 se reunieron Gontran Ervynck y David Tall para formar un grupo de estudio sobre Pensamiento Matemático Avanzado (PMA) y trabajar en un libro (Tall, 1991) con el mismo nombre, consideraron las matemáticas de secundaria y las de universidad, tratando de conectarlas con la manera de pensar de los matemáticos. Reorientaron la problemática del aprendizaje en términos de procesos cognitivos; las investigaciones abordaron tópicos que por su naturaleza y complejidad se situarían dentro de la llamada "matemática escolar superior" (límite, derivada, entre otros). Como producto de este trabajo en el año 2002 se publica el libro "ADVANCED MATHEMATICAL THINKING" editado por David Tall. 
Para Tall (1992) pasar del PME al PMA implica una transición que requiere una reconstrucción significativa. La reconstrucción se caracteriza por hechos trascendentes como pasar de describir a definir, pasar de convencerse a sí mismo a convencer a un amigo, pasar de convencer a un amigo a convencer a un enemigo; en otras palabras pasar de convencer a demostrar.

Por ejemplo Tall (1992) señala que el concepto de límite debe situarse dentro del PMA por los procesos cognitivos que son necesarios para su manejo. Cornu (1991) por su parte está de acuerdo en ello puesto que lo considera pieza fundamental en la teoría de las aproximaciones, continuidad, derivabilidad e integración. Entretanto Edwards, Dubinsky y McDonald (2005) indican que este concepto debe situarse en el PME o PMA dependiendo del trabajo que se realice con él. Si solamente se trabaja cálculo de límites, no se requiere el pensamiento matemático avanzado para realizar esta operación. Por su parte Claros, Sánchez y Coriat (2006) proponen un criterio para discriminar entre PME y PMA basado en los fenómenos descritos para las sucesiones con límite y las funciones con límite en un punto.

Una característica distintiva entre PMA y pensamiento elemental es la complejidad y la forma de abordarlos. Conceptos avanzados, tales como anillos o grupos de Lie, tienden a ser muy complejos. La diferencia radica en cómo esta complejidad es administrada. Los poderosos procesos son aquellos que permiten hacer esto, en particular, la abstracción y la representación. Por medio de la abstracción y la representación, uno puede pasar de un nivel de detalle a otro y, por lo tanto, gestionar la complejidad.

Un aspecto relevante es que los procesos matemáticos y psicológicos, en muchos casos son dos: los matemáticos y los aspectos psicológicos de un proceso y rara vez pueden ser separados. Por ejemplo, cuando se construye un gráfico de una función, se ejecuta un proceso matemático, siguiendo ciertas reglas que pueden ser expresadas en lenguaje matemático; sin embargo, al mismo tiempo, es muy probable generar una imagen mental visual del gráfico; en otras palabras, usted está visualizando la función en una forma que posteriormente puede ayudarle a razonar acerca de la función.

Las imágenes mentales y las matemáticas están estrechamente vinculadas. Tampoco puede surgir el uno sin el otro y en realidad son generados por el mismo proceso. Es precisamente este vínculo, lo que hace a estos procesos interesantes y relevantes para comprender el aprendizaje y el pensamiento en matemáticas avanzadas.

Hay muchas teorías competentes en psicología. Teorías comportamentales, construidas en observación externa de estímulos y respuestas, se rehúsan a especular acerca de trabajos internos de la mente. La psicología constructivista, por otro lado, intenta discutir como son creadas las ideas mentales en la cabeza de cada individuo. Esto podría plantear un problema dialectico para los matemáticos con el ideal platónico de las matemáticas existiendo independientemente de la mente humana, pero esto resulta ser una perspectiva significativa en los procesos creativos de investigación de los matemáticos así como las dificultades experimentadas por estudiantes matemáticos.

El gran psicólogo suizo Piaget vio la necesidad del individuo de hacer un equilibrio dinámico con su ambiente como un subyacente tema en su trabajo. Este equilibrio podría ser turbado a través de la confrontación con nuevo conocimiento que entrará posiblemente en conflicto con el antiguo. Así un periodo de transición podría ocurrir en el cual la estructura del conocimiento es reconstruida a un nivel más maduro de equilibrio.

Para Selden y Selden. (2013) se intenta hacer una distinción entre, por un lado, identificar directamente los tipos de pensamiento matemático que podrían considerarse avanzados a cualquier edad o nivel de grado, y por otro lado, tomar como avanzados los tipos de pensamiento característicos de los temas de matemática que podrían considerarse como avanzados. Por ejemplo, Edwards, et al. (1997) discuten esta distinción. En la práctica, sin embargo, estos dos puntos de vista distintos a menudo se respaldan entre sí. Es decir, para argumentar que 
algún tipo de pensamiento, como los hábitos de la mente y las habilidades asociadas con el manejo de la abstracción, es avanzado, se puede notar que dicho pensamiento a menudo ocurre al pensar en temas avanzados, como el álgebra abstracta. Por el contrario, si uno necesita argumentar que el álgebra abstracta es avanzada, se podría notar que gran parte del pensamiento involucrado es de hecho abstracto.

\section{Pensamiento matemático avanzado como proceso.}

Pensemos en un curso típico de matemáticas universitaria de primer año. El profesor tiene que cumplir con una gran cantidad de contenido relacionado con temas que involucran lo planificado para desarrollar en un periodo de tiempo, según el caso como un semestre académico. Por ejemplo sí es un curso de cálculo, álgebra, matemáticas finitas, métodos numéricos o de otro tipo, para el instructor el contenido a ser enseñado es conocido e irrefutable. Aunque el profesor probablemente piense en varias alternativas para organizar este material en una estructura limpia, lógicamente, cada una de estas estructuras, básicamente consisten en un número de teoremas, para ser probados y una serie de aplicaciones de estos teoremas a temas de matemáticas y más allá. El instructor posiblemente distribuye estos en tantos períodos de clases que están disponibles y charla durante una parte considerable de estos períodos de clases, haciendo un uso extensivo de sorprendentes y cómodos formalismos. Al hacerlo, un aspecto muy importante de las matemáticas que se enseña se presenta a los alumnos, a saber: el acabado y pulido producto en el que la bien conocida, irrefutable, plenamente aceptado segmentos de la matemáticas han crecido.

Esta forma de enseñanza tiene varias ventajas: por ejemplo, permite una estructura bien planificada del curso, así como el progreso predecible a través del material y, por lo tanto, bastante garantía de que la mayor parte del material incluido en el plan de estudios puede ser cubierto. Lamentablemente, también tiene al menos un grave inconveniente: es inflexible en términos de adaptabilidad a los estudiantes. También puede funcionar bastante bien para los estudiantes aventajados o talentosos. Pero como se muestra, por ejemplo, la actual crisis de cálculo, no funciona para la gran mayoría de los estudiantes, quienes en la especialidad de la ciencia, la ingeniería, la medicina o las artes requieren de la matemática como una disciplina del servicio.

Lo que los estudiantes aprenden, y no aprenden, está muy bien ilustrado por los resultados de diferentes estudios. En otras palabras, lo que la mayoría aprende en sus cursos de matemáticas, es a llevar a cabo un gran número de procedimientos normalizados, basados en formalismos para obtener respuestas claramente limitadas a unas clases de ejercicios. Terminan con una cantidad considerable metodología de trabajo del matemático pero sin conocimientos matemáticos, es decir, carecen de los conocimientos que les permita utilizar sus conocimientos de manera flexible para resolver los problemas de un tipo desconocido para ellos.

\section{Procesos involucrados en el desarrollo del pensamiento matemático avanzado}

Uno de los más importantes procesos en el desarrollo de este pensamiento es la abstracción que a su vez involucra procesos de representación, generalización, análisis y síntesis. A continuación se describe brevemente cada uno de ellos a partir de la caracterización presentada por Dreyfus (2002), en el capitulo Advanced Mathematical Thinking Processes, en el libro de David Tall.

\section{Procesos involucrados en la representación}

Las representaciones tienen una función muy importante en matemáticas: Si queremos hablar del grupo de permutaciones de $\mathrm{n}$ objetos, por ejemplo, a menudo será conveniente llamarlo el grupo simétrico de grado $\mathrm{n}$ y denota por Sn. La notación Snes un signo que se refiere, y por lo tanto representa o simboliza, el grupo en cuestión; es una representación simbólica del grupo. 
Los símbolos involucran las relaciones entre signos y significados; sirven para hacer que una persona reconozca el conocimiento implícito (el significado) explícito en términos de símbolos. Cuando hablamos o pensamos sobre un grupo, una integral, una aproximación, sobre cualquier objeto matemático o proceso a todos, a cada uno de nosotros se refiere a algo que tenemos en mente una representación mental del objeto o proceso bajo consideración.

Las respectivas representaciones mentales de la noción relacionada con un objeto matemático pueden ser muy diferentes. Para un estudiante la noción de una función puede ser limitada a los procesos de cálculo (o asignación), mientras que para el maestro las integrales indefinidas, se puede pensar de la función en la integral como un objeto a ser transformado. Una representación simbólica externa puede ser escrita o hablada, normalmente con el objetivo de hacer de la comunicación sobre el concepto más sencillo. Una representación mental, por otro lado, se refiere a esquemas internos o marcos de referencia que una persona utiliza para interactuar con el mundo externo.

Distintas representaciones mentales de un concepto pueden coexistir en la mente de alguien, $\mathrm{y}$ al ser bien aprovechadas pueden ser llamados a considerar diferentes situaciones matemáticas. Sin embargo, las diferentes representaciones mentales también pueden entrar en conflicto. Para tener éxito en las matemáticas, es deseable tener ricas representaciones mentales de los conceptos. Una representación es rica si contiene muchos aspectos vinculados de ese concepto. Es pobre si tiene muy pocos elementos para permitir flexibilidad en la solución de problemas. Aunque es importante tener muchas representaciones de un concepto, su existencia por sí sola no es suficiente para permitir un uso flexible del concepto de resolver un problema.

La visualización es un proceso por el cual las representaciones mentales pueden llegar a ser. Una descripción más general de cómo las representaciones mentales de los conceptos matemáticos pueden ser generados ha sido propuesto por Kaput(1987); de acuerdo a su teoría, el acto de generar una representación mental, se basa en los sistemas de representación, es decir, objetos concretos externos, que pueden ser materialmente realizadas. En el caso de funciones, los gráficos son uno de esos artefactos, fórmulas algebraicas son otra forma, diagramas y tablas de valor aún son otras.

Otro aspecto muy importante es el proceso de cambio de representaciones. Estrechamente asociada con la de representación. En nuestro contexto, significa ir a través de una representación de un concepto matemático a otro. Un enfoque posible es utilizar sistemáticamente varias representaciones en la enseñanza, destacando el proceso de cambio de las representaciones desde el principio. Un proceso que está estrechamente ligada a las representaciones de conmutación es la traducción. El significado de traducir lo que es relevante para el pensamiento matemático avanzado va a través de una formulación de declaración matemática o problema a otro. Por ejemplo, una ecuación diferencial lineal de segundo orden con coeficientes constantes pueden presentarse como un problema de oscilación, posiblemente con la fricción o rozamiento; su solución puede ser discutida en términos de miembros permanentes y transitorios.

El modelado como forma de representación. Normalmente, el término se refiere a encontrar una representación matemática de un objeto no matemático o proceso. En este caso, significa construir una estructura matemática o teoría que incorpora las características esenciales del objeto, sistema o proceso para ser descrito. Esta estructura o teoría, el modelo puede ser utilizado para estudiar el comportamiento del objeto o proceso que se modela. El proceso de la representación es, en cierta medida, análogo al proceso de modelado, pero en otro nivel. En la modelación de la situación o el sistema es físico y el modelo es matemático; en la representación del objeto a ser representado está la estructura matemática, y el modelo es una estructura mental 


\section{Los procesos involucrados en la abstracción}

Muchos de los procesos mencionados ocurren en cualquier nivel del pensamiento matemático. Sin duda, incluso los niños pequeños crean representaciones mentales de lo que piensan, y particularmente de los objetos matemáticos de pensamiento, tales como números o triángulos. Empezando a más tardar en la escuela primaria, los niños también trabajan con estos objetos, especialmente los números, en diferentes representaciones. Otros procesos, sin embargo, adquieren mayor importancia como los estudiantes desarrollan habilidades y experiencia matemática y como los contenidos matemáticos tratan de ser más avanzados; el más importante entre estos procesos avanzados es abstraer. Si el estudiante desarrolla la habilidad de hacer conscientemente abstracciones matemáticas de situaciones, él ha alcanzado un nivel avanzado de pensamiento matemático. El logro de esta capacidad para abstraer puede muy bien ser el único y más importante objetivo de la enseñanza de la matemática avanzada Dreyfus (2002).

El proceso de generalización. Generalizar es derivar o inducir a partir de datos, para identificar puntos comunes, para ampliar los dominios de validez. Un estudiante puede saber por experiencia que una ecuación lineal en una variable tiene una solución, y que la "mayoría" de los sistemas de dos (o tres) ecuaciones lineales en dos (o 3) variables tienen una solución única. Entonces puede generalizar para sistemas de $\mathrm{n}$ ecuaciones lineales en $n$ variables. Lo que es más importante, con una orientación adecuada, el sistema podrá ser llevado a examinar el significado de "más" para $n=2$ y n=3 en la declaración anterior, formular como una condición apropiada y generalizar también esa condición para $\mathrm{n}>3$. En este proceso, para hacer la transición de los casos particulares de $n=2,3,4$ en general $n$, se necesita identificar la condición para $n=2$ y $n=3$ qué tienen en común, y de la conjetura establecer luego el dominio de validez de la conclusión "no hay una solución única" puede extenderse a general n.
La generalización en el ejemplo anterior es importante en el sentido de que establece un resultado para una amplia clase de casos, todos los sistemas de $\mathrm{n}$ ecuaciones lineales independientes en $\mathrm{n}$ variables. Otras generalizaciones no incluyen la necesidad de tal concepto de formación. Un ejemplo es la transición de la convergencia de una secuencia de números para la convergencia de una secuencia de funciones, lo que da lugar a la necesidad de una topología en el espacio de las funciones. Las exigencias cognitivas en el proceso de generalización se ha incrementado considerablemente.

El proceso de síntesis. Sintetizar significa combinar o componer piezas de tal manera que forman un todo, una entidad. Todo esto, a menudo representa más que la suma de sus partes. Por ejemplo, enálgebra lineal, usualmente los estudiantes aprenden un gran número de hechos aislados sobre ortogonalización de vectores, diagonalización de matrices, la transformación de las bases, solución de sistemas de ecuaciones lineales, etc. Más adelante en el proceso de aprendizaje, todos estos hechos no relacionados anteriormente esperemos que se funden en una sola imagen, dentro de la cual todos ellos son compuesto e interrelacionados. Este proceso de fusionar en una sola imagen es una síntesis.

\section{La abstracción}

El proceso de abstracción, está íntimamente ligado a la generalización. Uno de los principales incentivos para la abstracción es la naturaleza general de los resultados que pueden obtenerse. Otro incentivo es el logro de la síntesis. Mostrar a grupos de estudiantes que es posible describir en una forma unificadora de un gran número de situaciones que hasta ahora han sido consideradas por separado e independientemente uno del otro. Pero ni generalizar ni sintetizar requieren las mismas exigencias cognitivas en estudiantes como abstracción. La generalización suele implicar una ampliación de la estructura de conocimiento del individuo, mientras que la abstracción es probable que implique una re-construcción mental. En la transición digamos, 
de los números reales a los números complejos, podamos lograr la generalización al no insistir más en el orden, pero seguimos trabajando con los objetos que están representados de forma explícita mediante números que podemos sumar y multiplicar en un medio familiar. La abstracción, sin embargo, exige que el estudiante debe centrarse en las relaciones que existen entre los números para poder entender que se trata de un campo, en lugar de los números mismos. Igualmente con otras nociones como la de función, el grupo y el espacio vectorial.

La abstracción, por lo tanto, contiene el potencial de generalización, la síntesis y viceversa, obtiene su objetivo principalmente de este potencial de generalización y síntesis. La naturaleza del proceso mental de abstracción es, sin embargo, muy diferente a la de generalización y síntesis. Abstraer es ante todo un proceso constructivo. La construcción de estructuras mentales, de estructuras matemáticas, es decir, de propiedades y relaciones entre objetos matemáticos. Este proceso depende del aislamiento de las propiedades y relaciones. Se requiere la capacidad para reorientar la atención de los propios objetos de la estructura de sus propiedades y relaciones. Esa actividad mental constructiva por parte de un estudiante depende en gran medida de la atención del estudiante. Está centrado en las estructuras que forman parte del concepto abstracto y apartando aquellos que son irrelevantes en el contexto; la estructura se vuelve importante, mientras se omite detalles irrelevantes, reduciendo la complejidad de la situación.

Para Dreyfus (2002) hay una dificultad inherente a la abstracción: ¿Cómo podemos generar estructuras mentales, que tan a menudo están vinculadas a imágenes visuales, si deben representar las relaciones que son retirados de los objetos concretos que originalmente estaban vinculadas? ¿Cuál es la función de visualización en el proceso de abstracción? De nuevo, no hay una respuesta clara a esta pregunta. Las imágenes visuales son generalmente globales y subrayan los aspectos estructurales. Por lo tanto, si las imágenes visuales apropiadas pueden ser encontradas, lo más probable es que sean de gran ayuda a los estudiantes en los procesos de abstracción.

Finalmente, representación y abstracción son procesos complementarios en direcciones opuestas: por un lado, un concepto a menudo se abstrae de varias de sus representaciones, por otro lado las representaciones son siempre representaciones de algún concepto más abstracto. Cuando una representación única de un concepto es utilizada, la atención puede centrarse en esto en lugar del objeto abstracto. Sin embargo, cuando varias representaciones están siendo consideradas en paralelo, la relación con el concepto abstracto subyacente se vuelve importante.

Hoffman (1989) citado Dreyfus (2002) propone una filosofía de la educación matemática basada en el simple reconocimiento de que "la matemática es una actividad humana". Siendo tarea de los profesores transmitir a sus estudiantes una imagen de las matemáticas como una ciencia que incorpora la observación, la experimentación y el descubrimiento. Para Garbin (2015) el "pensamiento matemático se desarrolla en todos los seres humanos en el enfrentamiento cotidiano en múltiples tareas que incluyen por un lado, pensamiento sobre tópicos matemáticos, y por otro, procesos avanzados del pensamiento como abstracción, justificación, visualización, estimación y razonamiento" (p. 139).

El conocimiento matemático es un proceso que ocurre en la mente del estudiante (Dreyfus, 2002). Para este autor los procesos matemáticos y psicológicos no pueden ser separados, tampoco puede surgir él uno sin el otro, debido a que las imágenes mentales y las matemáticas están estrechamente vinculadas y procesos de abstracción tienen como requisito los procesos de representación, generalización y síntesis.

Por todo lo anterior los profesores de matemáticas debemos aceptar y reconocer que los procesos de representación, generalización, síntesis y abstracción que contribuyen en el desarrollo del pensamiento matemático (ya sea PME o PMA) ocurren en la mente del estudiante. Pero no basta sólo con reconocer esto, debemos profundizar en 
ellos e integrarlos en la planificación de actividades para ser implementados dentro o fuera del aula, en ambientes computarizados o no, pero teniendo siempre presente que son procesos a desarrollar en la mente del estudiante y no desde el punto de vista tradicional del docente.

Por ejemplo el planear e implementar actividades que promuevan en el estudiante diferentes representaciones (tanto internas como externas) a partir de casos concretos, establecer relaciones y vínculos entre ellas, sí estas son lo suficientemente robustas pueden llevar a la generalización, síntesis y abstracción.

Otra alternativa la ofrecen Mason et al. (1988) en su libro titulado Pensar Matemáticamente. Basan su propuesta metodológica en la resolución de problemas, que permite el desarrollo del pensamiento matemático por medio de la sistematización y reflexión de este proceso en cada individuo. Los autores plantean tres fases en el proceso de resolución de problemas matemáticos: entrada, ataque y revisión. Ellos afirman que en el momento en el que un individuo haga explícita cada una de estas fases durante la resolución de cualquier problema de matemáticas, podrá tener herramientas que faciliten el desarrollo del pensamiento matemático (Gómez, 2009 ).

Posiblemente si llevamos estas actividades al aula de clase nuestros estudiantes percibirán de pronto que la representación, generalización, síntesis y abstracción son procesos están en sus mentes y por tanto son una actividad humana, no una actividad acabada e irrefutable que simplemente hay que reproducirla.

\section{Conclusiones}

Una dificultad común observada en los estudiantes que aprenden matemáticas avanzadas es su queja de que el tema es demasiado abstracto. Los términos "generalización" y "abstracción" se usan en matemáticas tanto para denotar procesos en los que los conceptos se ven en un contexto más amplio como también los productos de esos procesos. La abstracción es un objeto mental muy diferente, que se define por una lista de axiomas. Mientras que la generalización simplemente implica una extensión de procesos familiares, este último requiere una reorganización mental masiva. Cognitivamente no se trata sólo de un proceso de deducción, sino de un proceso de construcción en el que el alumno está construyendo propiedades del objeto abstracto.

El párrafo anterior es sólo una muestra sólo de una de las miles de dificultades a la que se ve enfrentado el estudiante sobre todo en niveles de educación universitaria. Cómo se aclaró el documento trata de dar una mirada del desarrollo del pensamiento matemático como proceso que ocurre en la mente y por tanto como una actividad humana. El documento se queda corto en su análisis. Por ejemplo hay que dar una mirada al papel que juega la intuición, la creatividad, el concepto, la imagen del concepto, la definición, la prueba o demostración, las dificultades, los obstáculos entres otros que forman parte del desarrollo del pensamiento matemático.

\section{Referencias}

Camarena G.P. (2009). La matemática en el contexto de las ciencias. Innovación Educativa, 9(46) 1525. Recuperado de https://dialnet.unirioja.es/ descarga/articulo/4707699.pdf

Claros, F. J., Sánchez, M. T. y Coriat, M. (2006). Fenómenos que organizan el límite. En P. Bolea, M. González y M. Moreno (Eds.), Investigación en Educación Matemática X, 157-171.

Cornu, B. (1991). Limits. En D. Tall (Ed.), Advanced Mathematical Thinking (pp. 153166). Dordrecht: Kluwer.

Dreyfus, T. (1990). Advanced Mathematical Thinking. In P. Nesher\& J. Kilpatrick (Eds.), Mathematics and Cognition: A Research Synthesis by the International Group for the Psychology of Mathematics Education (pp. 113134). Cambridge: Cambridge University Press. Dreyfus, T. (2002). Advanced mathematical thinking processes. In Advanced mathematical thinking (pp. 25-41). Springer Netherlands . 
Edwards, B. (1997). An undergraduate student's understanding and use of mathematical definitions in real analysis. In J. A. Dossey, J. O. Swafford, M. Parmantie, \& A. E.

Edwards, B. S., Dubinsky, E. y McDonald, M. (2005). Advanced mathematical thinking. Mathematical Thinking and Learning, 7(1), 1525.

Garbin, Sabrina (2015). Investigar en pensamiento matemático avanzado. En Ortiz, José; Iglesias, Martha(Eds.), Investigaciones en educación matemática. Aportes desde una unidad de investigación (pp. 137-153). Maracay, Venezuela: Universidad de Carabobo

Gómez, John (2009). La resolución de problemas en el pensamiento matemático avanzado: el caso de la elaboración de significados de la definición de espacio topológico. Comunicación presentada en $10^{\circ}$ Encuentro Colombiano de Matemática Educativa (8 a 10 de octubre 2009). Pasto, Colombia.

Kaput, J. J., (1987a), 'Towards a theory of symbol use in mathematics', in C. Janvier (Ed.) Problem sof representation in mathematics learning and problema solving, Erlbaum, Hillsdale NJ.

Mason, J., Burton, L. \& Stayce K. (1988). Pensar matemáticamente. (reimp. XVII). (Trad. Mariano Martínez P.) España: Editorial Labor S.A. (Original publicado en 1982).

National Council of Teachers of Mathematics (NCTM). (2000). Principles and Standards for School Mathematics. Reston, VA: The National Council of Teachers of Mathematics.

Ministerio de Educación Nacional. (2009). Educación Superior: competencias genéricas en educación superior. Boletín informativo (13). Recuperado el 31 de marzo de 2015 de: http://www.mineducacion.gov.co/1621/ articles-92779_archivo_pdf_Boletin13.pdf

Selden, A. \& Selden, J. (2013). Advanced mathematical thinking: A specialissue of mathematical thinking and learning. $10.4324 / 9781315045955$.

Sternberg, R. J. (1996). What is mathematical thinking? En R.J. Sternberg y Ben-Zeev (Eds.), Thenature of mathematical thinking (pp. 303318). Mahwah, NJ, USA: Lawrence Erlbaum. Tall, D. (2002). Advanced Mathematical Thinking (7aed.). Enlínea: Kluwer Academic Publishers 